\title{
Methodological challenges in design and conduct of randomised controlled trials in acupuncture
}

\author{
Yu-Tong Fei and colleagues examine the problems with designing and implementing trials of
}

\author{
acupuncture
}

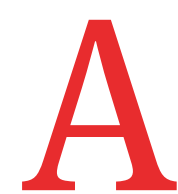

cupuncture is one of the most commonly used complementary and integrative therapies worldwide. During the past five years, results of over 4000 acupuncture trials have been published. As with other non-pharmacological interventions, challenges exist when designing and conducting randomised controlled trials in acupuncture. We discuss the most important challenges specific to acupuncture, some of which may apply to other nonpharmacological therapies. Detailed solutions to these challenges are discussed in another article in this series. ${ }^{1}$

\section{Simplified and poorly documented protocols}

Like surgery, behavioural therapy, and other non-pharmacological interventions, acupuncture treatment has a number of components. Although the selection of acupoints is usually documented in randomised controlled trials, ${ }^{2}$ other vital elements that acupuncturists consider equally important are often under-reported and oversimplified. ${ }^{3}$ These include acupuncture dose, length of needles, depth of insertion, number of needles, and manipulation

\section{KEY POINTS}

- Acupuncture is a complex intervention and when tested in trials is often oversimplified and poorly documented

- Acupuncture therapies are complex because of their multifaceted techniques, dependence on expertise, often combined therapies, and highly personalised treatment regimen

- Contextual effects, especially patientpractitioner interaction and participants' expectations, may impact the treatment effect of acupuncture and a trial's feasibility

- Sham acupuncture is difficult to implement and has a specific effect that might result in underestimation of the real acupuncture treatment effect after insertion; patient-doctor communication during the diagnostic and treatment process; expertise of acupuncturists; combination with other therapies; and personalisation of the intervention. ${ }^{45}$

\section{Complex intervention}

Methods to locate acupuncture points vary. In 2008, the World Health Organization regional office for the Western Pacific published a manual of standard acupuncture point locations, ${ }^{6}$ which has been well adopted. However, there are other acupoint location systems which vary somewhat from the WHO standards but are esteemed by specific schools of acupuncturists, such as Yang Jiasan's acupoint system. ${ }^{7}$

Acupuncture theory varies, contributing to differences in treatment regimens. Practitioners using traditional Chinese medicine theory to guide acupoint selection might prefer to use meridian based or symptom based acupoint selection methods. Western medical acupuncture bases its treatment on sensory stimulation. As an acute pain stimulus, acupuncture can elicit a euphoric feeling by increasing release of opioid peptides (such as endorphins and dynorphins), thereby alleviating ongoing pain. 8 Acupuncture stimulation serves the function of a stimulus and can achieve muscle reflex relaxation. 9 To treat musculoskeletal pain,10 most western practitioners in primary care, rheumatology, orthopaedic, and pain clinics will likely choose Ashi or trigger points (box 1).

Current evidence for acupoint specificity varies and complicates selection of treatment regimens. Acupoint specificity refers to different treatment effects that the selection of acupoints brings. A randomised controlled trial investigated acupuncture as an adjunct to antianginal drug therapy for chronic stable angina. The study showed that stimulating acupoints on the disease affected meridians resulted in four fewer angina attacks than stimulating acupoints on non-affected meridians (95\% confidence interval -2.43 to -5.71 ) during week 13 to $16 .{ }^{15}$ However, individual patient data meta-analysis on acupuncture for chronic pain showed no significant association between pain relief and the acupoint prescription (fixed, flexible, and individualised prescription). ${ }^{16}$

Acupuncture treatment dosage can differ significantly and impact the treatment effect. ${ }^{17}$ Duration, intensity, repetition intervals, and number of treatments and needles are important components of acupuncture dosage. Existing randomised trials of acupuncture have, however, either under-reported this critical information or used vastly different treatment dosages. For example, the latest Cochrane review ${ }^{18}$ addressing acupuncture for the treatment for depression found that only two thirds of the included randomised controlled trials reported needling duration, while in 63 included trials, treatment frequency varied from twice daily to once a week.

Existing evidence has explored the impact of acupuncture dose on treatment effect but rarely addressed the possible interaction between dose components. An individual patient data meta-analysis of acupuncture for chronic pain found a significant association between pain relief and the number of acupuncture treatment sessions but not de qi (box 1), frequency of sessions, duration of sessions, and number of needles used. ${ }^{16}$ Other studies found a larger number of needles, ${ }^{19}$ higher intensity, ${ }^{20}$ and greater frequency of treatment ${ }^{21}$ may increase treatment effects. For example, in a randomised controlled trial on knee arthritis, electroacupuncture $(>2 \mathrm{~mA})$ with a strong current relieved more pain. ${ }^{22}$ When designing randomised controlled trials in acupuncture, large variability and limited research evidence make it challenging for trialists to determine the appropriate treatment dosage.

Needle manipulation technique may affect the magnitude of the treatment effect. $^{23}$ The complex manipulation techniques include lifting, thrusting, twirling range, de qi sensation, and frequency of electrostimulation. Devices might help to detect and standardise the needle manipulation features but are not yet widely known and rarely used in trials. ${ }^{24}$ 
Box 1: Acupuncture terminology

- Acupuncture-Any type of intervention involving penetration of the skin with needles or stimulation of certain points with other methods regardless of its theoretical basis, excluding forms combined with moxibustion or medication such as warm needling, acupoint injection, or hydro-acupuncture ${ }^{11}$

- Ashi point-An acupuncture point with no specific name or definite location, the site of which is determined by tenderness or other pathological responses ${ }^{12}$

- De qi sensation-A sensation patients describe as pulling, numbness, heaviness, dullness, or aching that they experience during needling and which the acupuncturists might feel through sensations conveyed via the needles ${ }^{13}$

- Trigger point-A sensitive area of the body that produces a reaction elsewhere when stimulated ${ }^{12}$

- Manual acupuncture-The most commonly used type of acupuncture uses skin penetration with thin, solid, metallic needles manipulated by the hands ${ }^{12}$

- Electro-acupuncture-Additional electric stimulation following needle insertion ${ }^{12}$

- Syndrome pattern differentiation and its treatment-In Chinese medicine, it refers to the diagnosis based on a comprehensive analysis of symptoms to determine the cause, nature, and location of the illness. Even within the same disease, different syndrome patterns may dictate different treatments. When selecting an acupuncture treatment regimen, syndrome pattern differentiation can guide the meridian and acupoints selection. For instance, in two patients with upper respiratory infections caused by the same virus, the patient whose primary symptom is nasal congestion may have an important different syndrome pattern from that of a patient whose primary symptom is high fever. Acupoint selection can therefore be different for these patients ${ }^{12}$

- Meridians-Primary and collateral channels, regarded as a network of passages through which Qi (the vital life force in the body that acupuncture is designed to regulate) circulates and along which acupoints are distributed. Over 400 acupoints lie on 14 meridians over the human body

- Constitution-The characteristics of an individual, including structural and functional characteristics, temperament, adaptability to environmental changes, and disease susceptibility ${ }^{12}$

- Tube needle-A type of acupuncture delivery method that consists of two parts: a needle and a plastic tube. Practitioners will insert the needle into the skin through the plastic tube $e^{14}$

\section{Importance of expertise}

As a non-pharmacological intervention, the expertise of the practitioner who delivers acupuncture might influence its effectiveness. As recommended in the Revised Standards for Reporting Interventions in Clinical Trials of Acupuncture (STRICTA), ${ }^{25}$ trialists should report acupuncturists' expertise.

The evidence regarding the impact of expertise is, however, conflicting. An individual patient data meta-analysis based on acupuncture trials for pain conditions included 39 trials enrolling 20827 patients. Almost all participants $(97.3 \%)$ lived in western countries. Of the 39 trials, 14 (36\%) had no requirement for acupuncturists to have clinical experience, 20 trials (51\%) required between six months and four years' experience, and five trials (12.8\%) at least five years' clinical experience. Results revealed no apparent association between experience and the magnitude of the treatment effect. ${ }^{16}$ Between trials comparisons are, however, less robust than within trial comparisons, and a single trial not included in the meta-analysis reported that senior acupuncturists achieved a larger treatment effect than their juniors. ${ }^{26}$

\section{Used as combination therapy and highly personalised}

Acupuncture regimens often include conventional treatments and multiple acupoint stimulation therapies, such as manual or electro-acupuncture or body acupuncture plus auricular therapy. With regard to personalisation in determining treatment, the acupuncturist in traditional Chinese medicine considers the patient's syndrome pattern differentiation or meridian or body constitution (box 1). Patients with the same disease diagnosed by western medicine may show different syndrome patterns; acupuncture treatments vary accordingly. ${ }^{27}$

In clinical trials, many researchers prefer fixed acupuncture treatment protocols that neglect syndrome patterns or semi-fixed treatment protocols based on simplified pattern differentiation, rather than complex real world treatment packages of multiple acupuncture interventions, to reduce the difficulty of treatment quality control. This simplification of the intervention in clinical trials may provide only indirect evidence for treatments actually used in practice, possibly resulting in an underestimation of acupuncture's treatment effect as administered in (at least eastern) clinical practice.

\section{Trials often overlook contextual effects}

The context in which clinicians deliver healthcare interventions can improve or reduce the overall effect of an intervention. Like other non-pharmacological interventions, factors such as the patientpractitioner relationship and patients' expectations could impact acupuncture's effectiveness. $^{28}$

Importance of patient-practitioner interaction The process of establishing a confident and trusting relationship can be critical to an intervention's impact. ${ }^{429}$ Patients have identified a contrast in their relationship between western biomedicine practitioners and acupuncturists. ${ }^{4}$ Traditional Chinese medicine diagnostic and management procedures include listening to patients speaking about their health condition and attention to patients' feeling, mood, diet, rest, physical exercise, touching of the skin, and peaceful time spent together; each communicates that the clinician's knowledge of their particular experience is essential. The process tends to build a close relationship between acupuncturists and patients. ${ }^{30}$

\section{Participants' expectations can modify treatment effect}

Eight hundred and sixty four patients participating in four different trials investigating migraine, tension-type headache, chronic low back pain, and knee osteoarthritis were randomised to eight weeks of acupuncture treatment or sham acupuncture (superficial needling of non-acupoints). ${ }^{31}$ After adjustment for confounders including intervention group, age, sex, and duration of complaints, in comparison with baseline patients in both standard and sham acu- 
puncture groups with a high expectation of benefit proved more likely to achieve a reduction in pain of more than $50 \%$ than those with a low expectation (odds ratio of high to low expectation $1.67,95 \%$ confidence interval 1.20-2.32). ${ }^{31}$ Results are consistent with neuroscience research suggesting that anti-analgesic expectancy could block analgesia procedures and reduce pain relief. ${ }^{32}$ Trialists are increasingly making use of participants' expectations measures, such as the acupuncture expectancy scale, a validated instrument that measures the benefits patients perceive they would gain from acupuncture. ${ }^{33}$

Prior expectations towards acupuncture may also influence patient enrolment and retention in clinical trials. Patients who hold a strong belief in or against acupuncture treatment may decline participation, either afraid of being allocated to the sham group or unwilling to accept acupuncture treatment. If they do enter a trial, their prior beliefs may influence compliance and trial retention. ${ }^{34}$

\section{Sham acupuncture is problematic}

Aside from specific effects, an intervention may also have non-specific effects. Observation, assessment, and therapeutic rituals may elicit non-specific effects. ${ }^{35}$ Trialists often employ a placebo control with blinding of patients to differentiate the nonspecific effect of the target intervention. There are, however, challenges in using placebo control in acupuncture trials.

To achieve blinding, trialists have used many types of sham acupuncture. Sham acupuncture without skin penetration, such as the retractable blunt needle, is one of the earliest sham acupuncture inventions. ${ }^{36}$ Tube needles can hide the entire treatment process from view, but the procedure neither allows the angled insertion or manipulation technique after insertion nor applies to all acupoints (for instance, points on fingers and scalp). ${ }^{37}$ More importantly, it might not adequately blind the participants, particularly those who have experienced previous acupuncture treatment. ${ }^{38}$ Sham acupuncture may therefore aim to simulate the experience of optimal acupuncture more closely by more shallow insertion of needles in suitable acupoints; not undertaking subsequent manipulation; selecting acupoints in the relevant meridians but not specific to the health condition; selecting acupoints in irrelevant meridians; selecting skin areas close to but differing from the optimal acupoints; or some combination. ${ }^{3940}$ Though the acupuncturist will remain unblinded, this can blind patients more successfully.

However, a serious problem exists with sham acupuncture. Evidence suggests needle insertion or even skin touching creates specific effects. ${ }^{41}$ Thus, the effects of sham acupuncture may be similar tothough presumably lesser-optimally performed acupuncture. Additional evidence comes from the work of Harris et al, who found that acupuncture increases central $\mu$-opioid receptor binding potential, while sham acupuncture reduces or does not change it, ${ }^{42}$ and from the work of Zucker et al, who reported a larger pain reduction when using sham over real acupuncture in fibromyalgia patients with lower pain thresholds. ${ }^{43}$ Furthermore, specific effects of different types of sham stimulation may differ: penetrating needle sham, in comparison with other shams, showed the largest chronic pain relief. ${ }^{16}$ The crucial message from this work is that trials using sham acupuncture may underestimate acupuncture's treatment effect.

\section{Implications for future trials}

Acupuncture originated from a historical system of physiology, pathology, and treatment philosophy. However, the modern context requires alignment with current scientific methods, techniques, and findings. Modern basic science is now tackling the mechanisms of acupuncture effects. For example, the 2021 Nobel Prize research findings addressing piezo receptors for touch sensations ${ }^{41}$ may shed light on how penetrating or non-penetrating needling initiates human physical reaction. Current research priorities include investigating contextual effects, acupoint specificity, and the specific effect of sham acupuncture. When designing randomised controlled trials in acupuncture, trialists should consider the relevant components (as mentioned above) to determine the appropriate treatment dosage to achieve the optimal treatment effect. Organisations, in particular WHO, could involve prestigious academic societies and leading international acupuncture trialists to develop international guidance that facilitates development of acupuncture treatment protocol.

\section{Conclusions}

Randomised controlled trials of acupuncture face specific challenges. Firstly, acupuncture treatment protocols are often simplified and poorly documented with considerations that include acupuncture techniques varying in the underlying theory and thus methods to locate acupoints, treatment dosage and needle manipulation techniques, expertise of those delivering the acupuncture treatment, combination therapies, and, particularly in the eastern Asian countries, individualised treatment regimens. Secondly, contextual effects of treatment, including therapeutic patientpractitioner interaction and participants' expectations, are often overlooked. Thirdly, in view of the complex nature of the acupuncture intervention, sham acupuncture is both difficult to implement and might have specific effects, therefore underestimating the optimal treatment effect of acupuncture.

We thank Xiao-Li Li, Rui-Xue Hu, and Bing-Cong Zhao for providing references. Y-TF is supported by the National Natural Science Foundation of China (82074282). J-PL is supported by the Key Project of the National Natural Science Foundation of China (No 81830115).

Contributors and sources: YFei designed the paper's content and structure, formed the opinions, drafted and finalised the paper, and coordinated the research team. $\mathrm{H}$-JC participated in discussing the paper's structure, revised the table and main text, and organised the references. R-YX participated in discussing the structure of the paper, supported the content of the paper with selecting appropriate references, coordinated the literature process, and revised the manuscript. Q-YC participated in discussing the paper's structure and supported the content of the paper by selecting appropriate references. C-HL, YFeng, Y-RD, and M-KY participated in discussions, and revised versions of the manuscript. GG was involved in planning this series of papers and in extensively revising the manuscript. LT and $L-X L$ revised versions of the manuscript. J-PL designed the topic and structure of this paper, coordinated the research team, participated in discussions, and revised versions of the manuscript. $\mathrm{Y}$-QZ conceived the topic of this paper, coordinated the research team, participated in discussions, and was involved in revising the manuscript. All authors approved the final version of the manuscript. YFei, $\mathrm{H}$-JC $J-P L$, and $Y-Q Z$ contributed equally.

Competing interests: We have read and understood BMJ policy on declaration of interests and have no interests to declare.

Provenance and peer review: Commissioned; externally peer reviewed.

This article is part of a collection funded by the special purpose funds for the belt and road, China Academy of Chinese Medical Sciences, National Natural Science Foundation of China, the National Center for Complementary and Integrative Health, the Innovation Team and Talents Cultivation

Program of the National Administration of Traditional Chinese Medicine, the Special Project of "Lingnan Modernization of Traditional Chinese Medicine" of the 2019 Guangdong Key Research and Development Program, and the Project of First Class Universities and High-level Dual Discipline for Guangzhou University of Chinese Medicine. The BMJ commissioned, peer reviewed, edited, and made the decision to publish. Kamran Abbasi was the lead editor for The $B M J$. Yu-qing Zhang advised on commissioning for the collection, designed the topic of the series, and coordinated the author teams. Gordon Guyatt provided valuable advice and guidance.

Yu-Tong Fei, professor ${ }^{1,2,3}$

Hui-Juan Cao, associate professor $r^{1,2,3}$

Ru-Yu Xia, assistant professor ${ }^{1,2,3}$

Qian-Yun Chai, doctoral student ${ }^{1,2,3}$ 
Chang-Hao Liang, MSc student ${ }^{1,2,3}$

Yu-Ting Feng, MSc student ${ }^{1,2,3}$

Yi-Ran Du, MMed student ${ }^{1,4}$

Ming-Kun Yu, MSc student ${ }^{1,2,3}$

Gordon Guyatt, professor ${ }^{5,6}$

Lehana Thabane, professor ${ }^{5}$

Li-Xing Lao, professor ${ }^{7}$

Jian-Ping Liu, professor ${ }^{1,2,3}$

Yu-Qing Zhang, executive director ${ }^{5,8,9}$

${ }^{1}$ Centre for Evidence-Based Chinese Medicine, Beijing University of Chinese Medicine, Beijing, China

${ }^{2}$ Institute of Excellence in Evidence-Based Chinese

Medicine, Beijing University of Chinese Medicine, Beijing, China

\section{${ }^{3}$ Beijing GRADE Centre, Beijing, China}

${ }^{4}$ Dongzhimen Hospital, Beijing University of Chinese Medicine, Beijing, China

${ }^{5}$ Department of Health Research Methods, Evidence, and Impact, McMaster University, Ontario, Canada

${ }^{6}$ Department of Medicine, Faculty of Health Sciences,

McMaster University, Hamilton, Canada

${ }^{7}$ Virginia University of Integrative Medicine, Virginia, USA

${ }^{8}$ Ningbo GRADE Centre, University of Nottingham

Ningbo, China

${ }^{9}$ Chinese Academy of Medical Sciences, Beijing, China

Correspondence to: J-PLiu

Liujp@bucm.edu.cn

\section{(c) $\underset{\mathrm{BY}}{(1)}$ (8) OPEN ACCESS}

This is an Open Access article distributed in accordance with the Creative Commons Attribution Non Commercial (CC BY-NC 4.0) license, which permits others to distribute, remix, adapt, build upon this work non-commercially, and license their derivative works on different terms, provided the original work is properly cited and the use is non-commercial. See: http://creativecommons.org/ licenses/by-nc/4.0/.

\section{D) Check for updates}

1 Jiao R, Zang Y, Witt C, et al. A guide to clinical trialists: how to design high-quality acupuncture trials-a consensus informed by evidence. BMJ 2022.

2 Svenkerud S, MacPherson $\mathrm{H}$. The impact of STRICTA and CONSORT on reporting of randomised control trials of acupuncture: a systematic methodological evaluation. Acupunct Med 2018;36:349-57. doi:10.1136/acupmed-2017-011519

3 Liao XT, Ge SQ, Lu LM. [Thinking on applying expertise-based randomized controlled trials in acupuncture clinical research] [Chinese]. Zhonghua Zhongyiyao Zazhi 2020;35:4521-3.

4 MacPherson H, Thorpe L, Thomas K. Beyond needling--therapeutic processes in acupuncture care: a qualitative study nested within a low-back pain trial. J Altern Complement Med 2006;12:873-80. doi:10.1089/acm.2006.12.873

5 Loyeung BY, Cobbin DM. Investigating the effects of three needling parameters (manipulation, retention time, and insertion site) on needling sensation and pain profiles: a study of eight deep needling interventions. Evid Based Complement Alternat Med 2013;2013:136763. doi:10.1155/2013/136763

6 Regional WHO. Office for the Western Pacific. WHO standard acupuncture point locations in the Western Pacific Region. WPRO, 2008.

7 Liu QG. [Brief introduction of professor Yang Jia-san's academic thought] [Chinese]. Zhongguo Zheniiu 2008;28:359-64.
8 Han JS. Acupuncture and endorphins. Neurosci Lett 2004;361:258-61. doi:10.1016/j. neulet.2003.12.019

9 Melzack R. Myofascial trigger points: relation to acupuncture and mechanisms of pain. Arch Phys Med Rehabil 1981;62:114-7.

10 British Medical Association. Acupuncture: efficacy, safety and practice. Harwood Academic Publishers, 2000.

11 World Health Organization. Acupuncture: review and analysis of reports on controlled clinical trials. World Health Organization, 2003.

12 WHO international standard terminologies on traditional medicine in the Western Pacific Region. 2007. http://apps.who.int/iris/ handle/10665/206952

13 Zhu SP, Luo L, Zhang L, et al. Acupuncture De-qi: from characterization to underlying mechanism. Evid Based Complement Alternat Med 2013;2013:518784. doi:10.1155/2013/518784

14 Zhang CS, Zhang AL, Xue CC, Xie YM. New approach to preventing long acupuncture needles from buckling and contamination during insertion. Acupunct Med 2014;32:520-2. doi:10.1136/ acupmed-2014-010671

15 Zhao L, Li D, Zheng H, et al. Acupuncture as adjunctive therapy for chronic stable angina: a randomized clinical trial. JAMA Intern Med 2019;179:1388-97. doi:10.1001/jamainternmed.2019.2407

16 Vickers AJ, Vertosick EA, Lewith G, et al, Acupuncture Trialists' Collaboration. Acupuncture for chronic pain: update of an individual patient data metaanalysis. J Pain 2018;19:455-74. doi:10.1016/j. jpain.2017.11.005

17 White A, Cummings M, Barlas P, et al. Defining an adequate dose of acupuncture using a neurophysiological approach--a narrative review of the literature. Acupunct Med 2008;26:111-20 doi:10.1136/aim.26.2.111

18 Smith CA, Armour M, Lee MS, Wang LQ, Hay PJ. Acupuncture for depression. Cochrane Database Syst Rev 2018;3:CD004046.

19 Sun N, Tu JF, Lin LL, et al. Correlation between acupuncture dose and effectiveness in the treatment of knee osteoarthritis: a systematic review. Acupunct Med 2019;37:261-7. doi:10.1136/ acupmed-2017-011608

20 Lin LL, Tu JF, Wang LQ, et al. Acupuncture of different treatment frequencies in knee osteoarthritis: a pilot randomised controlled trial. Pain 2020;161:2532-8. doi:10.1097/j.pain.0000000000001940

21 Liu S, Wang Z, Su Y, et al. A neuroanatomical basis for electroacupuncture to drive the vagal-adrenal axis. Nature 2021;598:641-5. doi:10.1038/s41586021-04001-4

22 Lv ZT, Shen LL, Zhu B, et al. Effects of intensity of electroacupuncture on chronic pain in patients with knee osteoarthritis: a randomized controlled trial. Arthritis Res Ther 2019;21:120. doi:10.1186/ s13075-019-1899-6

23 Choi YJ, Lee JE, Moon WK, Cho SH. Does the effect of acupuncture depend on needling sensation and manipulation?Complement Ther Med 2013;21:20714. doi:10.1016/j.ctim.2012.12.009

24 Wang CC, Chai QY, Ren J. [Overview of techniques and methods for objective measurement of acupuncture stimulation] [Chinese]. Clin Med (Northfield III) $2014 ; 21: 32-4$

25 MacPherson H, Altman DG, Hammerschlag R, et al, STRICTA Revision Group. Revised STandards for Reporting Interventions in Clinical Trials of Acupuncture (STRICTA): extending the CONSORT statement. PLoS Med 2010;7:e1000261. doi:10.1371/journal.pmed.1000261

26 Wang CC. [A randomized crossover trial and methodology discussion on evaluating the effects of acupuncture manipulation by acupuncture doctors with different qualifications on the efficacy] [Chinese]. Beijing University of Chinese Medicine, 2016.

27 Ni L, Li F. [Application of evidence-based medicine in clinical efficacy evaluation of traditional
Chinese medicine] [Chinese]. Shanxi. / Tradit Chin Med 2008;10:57-8.

28 Bishop F, Al-Abbadey M, Roberts L, et al. Direct and mediated effects of treatment context on low back pain outcome: a prospective cohort study. BMJ Open 2021;11:e044831. doi:10.1136/ bmjopen-2020-044831

29 Paterson C, Britten N. Acupuncture as a complex intervention: a holistic model. J Altern Complement Med 2004;10:791-801. doi:10.1089/ acm.2004.10.791

30 Chae $\mathrm{Y}$, Olausson $\mathrm{H}$. The role of touch in acupuncture treatment. Acupunct Med 2017;35:148-52. doi:10.1136/acupmed-2016-011178

31 Linde K, Witt CM, Streng A, et al. The impact of patient expectations on outcomes in four randomized controlled trials of acupuncture in patients with chronic pain. Pain 2007;128:264-71. doi:10.1016/j.pain.2006.12.006

32 Goffaux P, Redmond WJ, Rainville P, Marchand S. Descending analgesia-when the spine echoes what the brain expects. Pain 2007;130:137-43. doi:10.1016/j.pain.2006.11.011

33 Mao JJ, Xie SX, Bowman MA. Uncovering the expectancy effect: the validation of the acupuncture expectancy scale. Altern Ther Health Med 2010;16:22-7.

$34 \mathrm{CaO} \mathrm{HJ}$, LiX, Li XL, et al. Factors influencing participant compliance in acupuncture trials: An in-depth interview study. PLoS One 2020;15:e0231780. doi:10.1371/journal. pone.0231780

35 Kaptchuk TJ, Kelley JM, Conboy LA, et al. Components of placebo effect: randomised controlled trial in patients with irritable bowel syndrome. BM/ 2008;336:999-1003. doi:10.1136/ bmj.39524.439618.25

36 Streitberger K, Kleinhenz J. Introducing a placebo needle into acupuncture research. Lancet 1998;352:364-5. doi:10.1016/S01406736(97)10471-8

37 Takakura N, Yajima H. A double-blind placebo needle for acupuncture research. BMC Complement Altern Med 2007;7:31. doi:10.1186/1472-6882-7-31

38 Takakura N, Takayama M, Kawase A, Yajima H. Double blinding with a new placebo needle: a validation study on participant blinding Acupunct Med 2011;29:203-7. doi:10.1136/ aim.2010.002857

39 Hershman DL, Unger JM, Greenlee H, et al. Effect of acupuncture vs sham acupuncture or waitlist control on joint pain related to aromatase inhibitors among women with early-stage breast cancer: a randomized clinical trial. JAMA 2018;320:167-76. doi:10.1001/ jama.2018.8907

40 Seca S, Patrício M, Kirch S, Franconi G, Cabrita AS, Greten HJ. Effectiveness of acupuncture on pain, functional disability, and quality of life in rheumatoid arthritis of the hand: results of a double-blind randomized clinical trial. J Altern Complement Med 2019;25:86-97. doi:10.1089/ acm.2018.0297

41 The Nobel Prize in Physiology or Medicine. 2021. Press release, 4 Oct 2021. https://www.nobelprize. org/prizes/medicine/2021/press-release/

42 Harris RE, Zubieta JK, Scott DJ, Napadow V, Gracely $\mathrm{RH}$, Clauw DJ. Traditional Chinese acupuncture and placebo (sham) acupuncture are differentiated by their effects on mu-opioid receptors (MORs). Neuroimage 2009;47:1077-85. doi:10.1016/j. neuroimage.2009.05.083

43 Zucker NA, Tsodikov A, Mist SD, Cina S, Napadow $\mathrm{V}$, Harris RE. Evoked pressure pain sensitivity is associated with differential analgesic response to verum and sham acupuncture in fibromyalgia. Pain Med 2017:18:1582-92 doi:10.1093/pm/pnx001

Cite this as: $B M J$ 2022;376:e064345

http://dx.doi.org/10.1136/bmj-2021-064345 